





\title{
DELLA RADICE
}

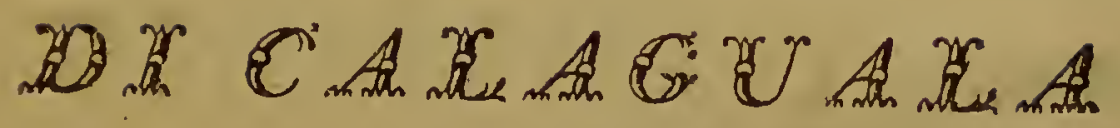
MEMORI A

\author{
$D I$
}

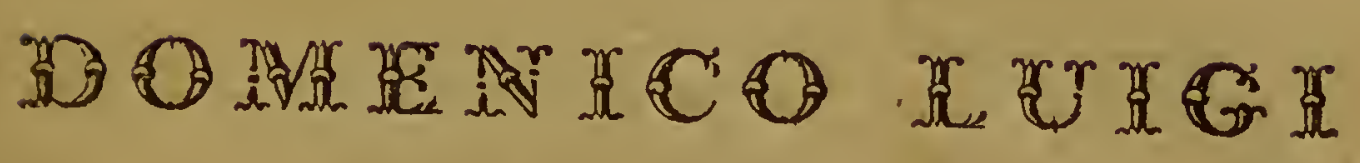

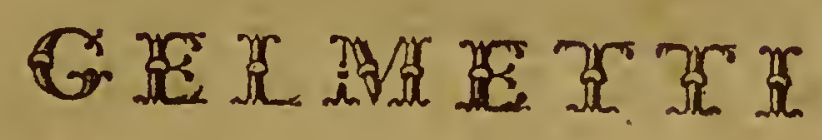

DOTTORE IN MEDICINA.

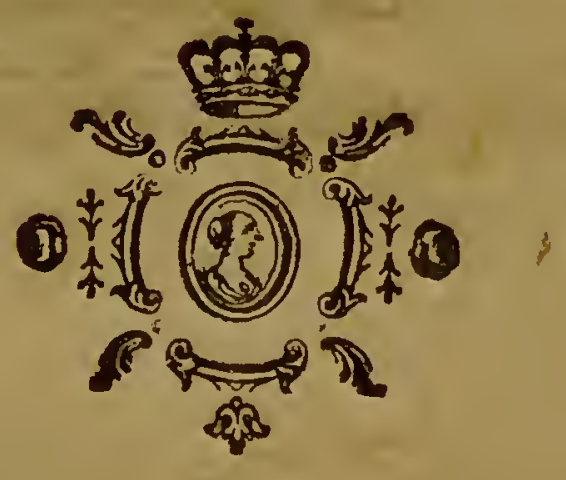

MA N TOVA.

Nilia Stamperia di Giuseppe Braglia.

$$
\begin{gathered}
\text { Con Permiss: } \\
\text { I } 788 .
\end{gathered}
$$


C'est le bazard qui nous a fait faire quelques décosvertes importantes, relatives à la cure des maladies; ce sont les natifs de l'Amérique \&o d'autres nations illitéreés, qui nous ont consmuniqué quelques remédes salutaires; .......

Grégory. Discours trad. de l'Angle par M. Verlac. Paris 1788 . 


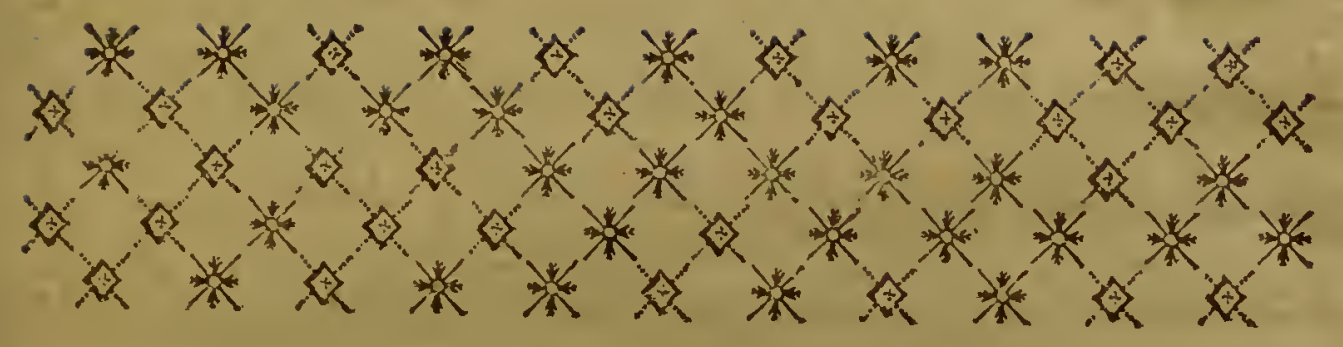

S. l' uso della radice di Calaguala utilissimo in diverse, e gravi malattie non è una sco. perta per l'Europa, è tale almeno per la nostra Lombardìa, e singolarmente per la città di Matitova. Meritava dunque il pensiere di qualche Cittadino, che la fama divulgasse del nuovo rimedio, il metodo additasse di prepararlo, e i casi, ove generalmente può convenire. Al Medico più che ad altri per dover d'istituto l'incombenza correva, ed a colui specialmente, che partecipe della cognizion della cosa, ed avendo su di essa vari sperimenti tentati, fosse nel caso di darne alcuna precisa idea. Or sono oltre quindici anni, che il Chiarissimo Sig. D. G. B. Borsieri già mio Precettore di Clinica nella R. Uni- 
4

versità di Pavia ( I ) premuroso, com' egli cra, d'istruire i suoi Allievi di tutto ciò, che i pro. gressi della Medicina concerne, comunicò la descrizione della Calaguala appoggiata alle scarse notizie, ch' egli ebbe dalla Spagna appena dopo l'iniroduzione della medesima in quel Regno. Ne conservai la memoria, e già mi cadeva in obblio, se lo strepito delle guarigio. ni colà avvenute non avesse impegnata la sensibilità d'alcuni illustri Personaggi a procurare anche alla Patria nostra il rimedio e il metodo più comune di praticarlo (2). Co-

( I ) Non posso rammentàre il nome di un Uomo, che alle qualità morali univa in grado eroico quelle di Filosofo, di Letterato, di vero Medico, senza provare i più teneri sentimenti di venerazione, di riconoscenza, e di dolore per la prematura, ed irreparabile sua perdita.

(2) Sono già parechj anni, che S. E. il Sig. Ball Gaetano Valenti Gonzaga Ricevitore di Malta in Milano, ed Ajo de' Principi Figliuoli delle LL. AA. RR. i Serenissimi Arciduchi Ferdinando d'Austria e Maria Reatrice D'Este, diede in dono una piccola porzione di radice di $C_{r}$. laguala alla Degnissima Lui Nipote Signora Contessa Don. na Marianna Guerrieri Zanardi della Virgiliana, qual ri- 
dest' occasione mi presento il modo di fare alcune osservazioni, le quali, previa l'accennata descrizione della pianta, ed unite a qualche nia riflessione, formano la tenue memoria, che cffro di buona voglia al Pubblico, perche vi ha diritto (3). Se dovrà soffrire qualche contraddizione, correria la sorte di qualunque novità. S'ella servirà di eccitamento

medio per le contusioni interne. Questa Ragguardevole Dama piena di sentimento d' umanità ne procurò maggior copia dalla Spagna, e di là assicurata dell'altre virtù dell' esotic radice, l'ha-generosamente distribuita giusta l'opportunità de'càsi, ed há avuro la grata compiacenza di va der coronate dall' esito il più felice molte malattìe, ch' erano state giudicate insuperabili.

In simile umanissima opera si è del pari prestato anche il Celebre Sig. Abate D. Giovanni Andres già abbastanza noto alla Repubblica Letteraria.

(3) 11 Chiarissimo Sig. Dottor Giammaria Mazzi Medic' ordinario dello Spedal grande di Milano antico mio amico e collega ricevette un mese fa queste medesime osservazioni, le quali, siccome mi avvertì, intendeva onorar le della stampa, facendole inserire nel Giornale Veneto di Medicina. Questo bastava per parteciparne alcuni della Professione, ma non già per facilitare, e rendere universale anche tra il popolo l' uso del predicato rimedio. 
per moltiplicare le sperienze intorno un rimedio, che tanto bene promette alla languente umanità, io sarò abbastanza riconpensato.

$$
\begin{gathered}
\text { D E S C I I I O N E } \\
\text { DELLA CALAGUALA. }
\end{gathered}
$$

Wel Regno di Quito dell' America Meridionale nasce una Pianta volgarmente appallata Calaguala. Questa ha le fuglie simili alla Lingua Cervina (Asplenium Scolopendirum Linn.); ma più brevi, più anguste, e termirate in purta più acuta. Esse pure sono più consistenti, e sugose. Nella loro parte supariore biancheggiano, nell'inferiore sono verdoscure, ed a questa sono aderenti i semi in certe masse lentiformi collocati, che formano un ordinata serie sì a destra che a sinistra. I semi staccansi dalle foglie senza lacerazione; al contrario di quelli della Lingua Cervina, che sono disposti a canto il nervetto di mezzo in linee oblique convergenti. La radice, 
da cui nascono le foglie, è di mezzana grossezza, ed a guisa della radice di Polipodio, va serpendo sotterra. Tagliata in pezzetti, nel colore, nella forma, nella leggerezza, e nelle fibre, o radicole, che sortono lateralmente, si rassomiglia moltissimo alla radice dell' Osmunda Regale. Trovasi d'ordinario sui monti di mezzan' altezza fra sassi, e ne' luoghi più sterili.

Questa radice sebbene non abbia un manifesto sapore (4), nullameno è potentemenre aperitiva, attenuante, e risolvente, com' esser sogliono, ma in grado minore, tutte le piante dorsifere. Adoprasi quindi comunemente dagl' Indiani nelle pleuritidi, nelle contusioni, e nelle aposteme interne.

\section{Il metodo di usarla è il seguente. Due}

(4) Quando è recente, come poco fa ebbi l'occasione di assaggiarne di quella pervenuta appena dalla Spagna, ha un odore e sapore leggermente aromatico, e lungamente masticata promove la salivazione. 
dramme di radice $s$ infondono in sufficiente quantità d'acqua di fonte ( $s$ ), e si fa bollire, finchè ne sia consumata la quarta parte. Di questo decotto se ne porgono a bere alcune tazze nel modo, che in Europa si usa la bevanda Thè. Non succede calore di so:te, nè veruna sensibile commozione, o evacuazione, toltane la copia delle urine, le quali passano con tanta veemenza, che alcu. na volta è sembrato ad esse unito un sangue disciolto. Contuttociò non si prova ardore o dølore nè ai lombi nè alle vie urinarie (6).

Giova finalmente sapere, che avvi un

(5) Alla dose di due dramme di radice unisco una libra e mezzo d'acqua, e così in proporzione, e fatta bollire in vaso chiuso, o cid che torna meglio, a bagno-marìa sino alla consumazione del terzo, ottengo un decotto ben saturato ed efficace.

(6) La stessa radice, che ha servito per la prima de. cozione, si può ribollire la seconda sino alla quarta volta, e si ha un decotto secondo, che si usa tra giorno, quando principalmente si desidera favorire una crisi già dichiatata salutare. 
altra specie di Calaguala, che chiamano fem. mina; e questa è di foglie larghissime, e lunghe, ma prive di semi, e credesi del tutto inurila. Uo altra radice parimenti si trova, che dicorio Calagualafalsa, la quale non ha di comune con la vera, che la sola radice. Questa per frode viene alle volte sostituita alla vera; ma rion è di veruna efficacia. La Calaguala vera si distingue per le sue foglie, che sono lunghe mezzo piede circa, larghe due dita, e riccamente fornite di semi, come sono a un dipresso le foglie dell'Emiontite (Asplenium Hemiontiris Linn.), e della Lingua Cervina.

\section{OSSERVAZIONE PRIMA.}

Il Sig. Gaetano Storoli Zanolini delia parrocchia di S. Appolonia di Mantova giovane vigoroso d'anni diciotto per irreparabil caduta in un pozzo avvenutagli, saranno tre anni, riportò uaa sì forte contusione al patA 5 
to, he oltre al tumore e alla lividura esterna, poco dopo gli si rese difficile il respiro, fu assalito da febbre con trisse molesta, sputi tinti di sangue, e dolore pungente al manco lato verso le coste superiori a guisa di pleuritide. Furongli prestati alcuni sussidj, e sembrò dopo pochi giorni assai sollevato. Tuttavia non cessava di lagnarsi del dolor laterale più, o meno acuto; il respiro era alquanto difficile, percosso colla mano il lato affetto, rispondeva di un suono cupo, e sordo, accoppiavansi di sovente certi rigori febbrili, continuava la tasse, e decadeva giornalmente di forze, e di nutrizione. Passò egl' intorno dus mesi in uno stato minacciante una vomica $e$ per conseguenza una tabe polmonare, quando fu trasferito a Gazuolo castello del mantovano, luogo in quel tempo di mia residenza, per sentire il mio parere. Esaminato attentamente il caso, ed avuto riflesso ai molti rimedj fin allora inutilmente tentati, mi 
risovenni delle celebrate facoltà della radice di Calaguala ne' casi anche jnuoltrati di contufione, e di aposteme interne. Ne consigliai pertanto l'uso, e per buona ventura si trovó la ricercata radice nella Farmacia di S. A. R. l' InFANTE di Parma. Fu quindi praticata in forma di decozione col metodo sovraccennato pel corso di giorni quaranta con i seguenii succeffi. Si resero da principio le orine più abbondanti, il colore diminuiva sensibilmente, il respiro era più facile, nè più si fecero sentire le solite febbrili alterazioni: Passati venti giorni, le orine deponevano un sedimento lodevole, e latriginuso. Ritornò in seguito l' appetito, il riposo, e le forze: Cessò la tosse, il respiro traevasi senza difficolta in ogni giacitura; tutto in fine si rimise allo stato naturale, anziché giunto fosse

il prescritto termine della cura, ed il suddetto Soggetto trovasi ora molto ben nodrito, vigoroso, e sano. 


\section{OSSERVAZIONE SECONDA.}

Il Sacerdote Sig. D. Giam-Battista Madella della parrecchia di Canicossa terra del mantovano, d' anni sessanta circa, d'alta statura, di tempra robusta, soffrì con pericolo nell' inverno dell' anno p. p. I787. di pleusifia giudicata in decimaquarta con una crisi di esperrorazione men che perferta. Fu quindi molestato di quando in quando dall'antico. dolor laterale e dal respiro alquanto grave ed affannoso. Nel gennajo del corrente i788. espostosi egli lungamente sotto il sole cocente contro il solito della rigida stagione, frese una infreddatura di petto, per la quale si suscitò una tosse incessante, con febbre, e il dolore si rese più acuto. Furongli prestati molti rimedj, ma senza notabil vantaggio; anz' insistendo in uno stato ambiguo sino a primavera innoltrata, l' Infermo si trasferì a Mantova sul finir d'aprile, ove fu da me vi: 
sitato. Trovai pertanto 'ch' egli era oppresso da febbre lenta abituale, da rosse con isputi rari marciosi, da dolore ot?uso, che dal sinistro lato estendevasi virso lo sterno: il decubito era difficile, e dolentissimo sulla parte affetta, aggiugnevansi il calor murdace della palma della mano, la macie di turto il corpo, la difficoltà dal respiro, la veglia, i sudori notturni, l'estenuazion delle forze. $D_{2}$ tali sintomi era facile l'argomentare il pessimo stato del polmone sinistro, e giudicare di un' attua! consunzione. Ponderate le rimote cagioni del morbo riposi ogni mia lusinga nell' azione prodigiosa della Calaguala: onde ne prescrissi il decotto da prendersi ben saturato mattina, e sera. Non furon vane le mie speranze, mentre l' Infermo resticuitosi per suo maggior comodo in villa, pochi giorni dopo mi scrisse ne' precisi termini seguenti. Egli è notabilissimo il miglioramento, che provo circa. il mio male, quantunque non fiero che sette $i$ 
4

giorni, dacbed pratico il docotto della connota radice. Se cosi prosiegue, come spero, la mia sotal guarigione firà prestissima. In ora la tos. se, ed il catarro pocbissimo mi molestano, le for. ze si fanno piiv robuste, Gormo assai bene senza essere dalla tosse angustiato, e molic bene mi riposo ancbia sopra il lato offeso. Proseguencio in meglio, si è ripristinato in salute in meno di trenta giorni, ed io ho avuto il piacere di vederlo perfettamente guarito e d'intendere da esso lui, come il rimedic non gli aveva arrecato il menomo incomodo, e ció ch'è più, non s' è mai avv=duto di alcuna sen. sibile evacuazione, o alterazione delle natu. rali secrezioni.

\section{OSSERVAZIONE TERZA:}

Giuseppe Lupi della parrocchia di S. Barnaba di Mantova d'anni ventisei, di professione Muratore, forre di costituzione, effendosi lungamente esposto a vento freddissimo 
dopo un violento esercizio della persona, fu 'nel cadere d' aprile di guess' anno assálito da. febbre con pungente, e fisso dolore tra la quine. ta, e sesta costa vera del destro lato. Uuironsi i rigori universali, la difincoltà del respiro, la tosse, l'oppressione di capo; e fu co. stretto giacere perpetuamente supino. Visitato nel secondo giorno di sua malattia, lo rinvenni con febbre acutiffima, i polsi erano bassi, ineguali, e duri, respirava a stento, nè poteva un momento contenersi dai dolorosi urti di rosse qualche volta seguita da sputi tenaci, e sanguigni. La faccia era rigonfia, e tinta di un rossu livido, gli occhi erano protuberanti, e torvi, aspra la lingua, e contratta, la sete inestinguibile. L'Infermo accusava un' interno ardore, ed oppreffione ai precordj: il ventre era teso, e renitente, le orine scarse e sanguigne, la pelle aspra e secca, e stavasi ansante giacendo qual immobile tronco. Si trassero in poche ore $\mathrm{da}$ trent' once di 
sangue, furono applicate fomenta al petto, ed alle inferiori estremià, e rdccomandate frequenti bevande di decozion d'orzo con ossimiele, e nitro. Sembiò calmata nel di seguenta l'infiammazione; ma risacerbatasi in quarta, si riapri la vena, e fu prescritta una mistura refrigerante, ed aperitiva. Nella quinta giornata gli sputi furono totalmente soppressi, la tosse era meno frequente, sentivasi alla gola un rantolo soffocativo, i polsi erano minuti, celeri, ed irregolari, soffermate le orine, ed un freddo sudore pioveva dalla fronte, e dal petto. La notre si passò in ismanie, in ansieta, in vaniloquj. La mattina di buon ora vidi l'Infermo con aspetto cadaverico, i polsi erano esili, ineguali, ed oscuri, il respiro sublime, ribollente il catarro, tutto in una $\mathrm{pa-}$ rola indicava assai vicina la morte. Pensai per azzardo alla radice di Calaguala, e ne prescrissi la decozione saturatissima. Senz'altro cibo o bevanda gli si fece ingojare, sebbene 
a siento, a cucchiai per tutto quel giorno senza notabile mutazion dé sintomi. Verso la notte si palesò un madore universale, le orine si resero più di frequente ed in copia maggiore, e si ottennero alcuni sputi di un catarro variegato tenacissimo. La mattina della settima ș̀ provò una calma notabile de' sintomi più minacciosi, i polsi erano più ordinati ed estesi, ravvivate le forze, e più facile la respirazione. Si continuò con più coraggio l' uso del rimedio, e continuarono pure $\mathrm{i}$ suoi buoni effetti a segno, che la mattina della nona il Malato sentì qualche sorta di appetito. $\mathrm{Ne}$ seguenti giorni si limitò il decotto a sole quattr' oncie di sei in sei ore fino alla perfetta convalescenza, che segui al compire della decima-quarta. Benchè la crisi di questa malattía non sia stata molto sensibile, è pero vero, che codest'Uomo al presente non prova dolore alcuno, difficoltà di respiro, o tosse, ma gode della migliore salute, e robustezza. 


\section{OSSERVAZIONE QUARTA.}

La Signora N. N. d' anni ventisette di gracile, e dilicata struttura, sogg tta a frequen. ti attacchi di asma convulfivo prodotto, come da replicate osservazioni si è poruto congetrurare, da un acido generantesi nello sto. maco, fu nello scorso maggio" assalita da fiera colica convulsiva con febbre, vomiti continui biliosi, e persinace stisichezza di ventre. Fu da principio preservata dal minacciato stato infiammatorio con cinque salassi, colle fomenta, e coi clistieri refrigeranti oleosi. Nulla peraltro giovò a calmare il vomiro, o i dolori crudeli, che dal manco Ipocondrio varso il Piloro estendevaufi con pungenti traficture. A questi sintoni si unirono il singlazozzo, gli sfinimenti, nè poteva contenere alimento, bevanda, o rimedio veruno. Era l' undicesimo giorno di si periglioso stato, quanso presentatomi il decotto di Calaguala a tut- 
i' altr' oggetto destinato, ne porsi all' Inferma alcuni sorsi a bere. Siccome non seguì il solito vomito, continuai ad esibirgliene di sovente, talchè in meno d' un' ora, n'aveva gì oltre una libra sorbito. Due ore appresso mitigaronsi i dolori, il vomito, il singhiozzo, e cominciò un sudore a manifesiarsi copioso alle gambe, che si diffuse poi al corpo tutto. Continuò la sensibile traspirazione per tutta la seguente notte, che fu tranquilla, e si ottennero eziandio diverse sedi di copiose e ben concotte materie. La mattinà si trovò $\mathrm{l}^{\prime}$ Ammalata sollevatissima, e proseguendo coll' uso del rimedio anche la declinazione del male, si condusse in pochi giorni fuor di pericolo. Alle accennate osservazioni aggiungo di essere assicurato del felice esito di una tisichezza incipiente, di una pleurisia, e recentissimamente di una polmonia catarrale coll' uso di questo stesso rimedio: casi tutti accaduti quì in Mantova, la descrizione de' 
quali tralascio, perche da me inosservati: RIFLESSIONI.

Veramente le riferite osservazioni non sono nè abbastanza numerose, nè affatto esatte per assicurarci delle specifiche virù della Calaguala; ma, come ognun vede, la loro precisione può dar valore a delle giuste lusinghe, e corágio ad ulteriori sperimenti. Se \& lecito argomentare dai risultati delle poche mie sperienze, sembra assai verosimile, che la detta radice abbia una facoltà risolventissima, corroborante, antispasmodica. Oltreciò determina colla maggiore piacevolezza, e sol. lecitudine le morbose ed eterogenee materie a segregarsi dal corpo umano, mediante l'aumento delle secrezioni, ed escrezioni, senza notabile, o pericoloso perturbamento dell' animale economia. Sembra infatti innegabile la sua risolvente facoltà nelle congestioni catarrali (osserv. 2., e 3.), e sanguigne (osserv. I.); 
Percio si potrà usare con sicurezza e vantaggio ne'mali di petto, che nascono per via di congestione molto a noi famigliari (7), nelle pertinaci infreddature, nelle tossi ribelli, nelle tisichezze specialmente pituitose, nelle contusioni, ne' reumi, nelle spurie, ed anche nelle vere infiammazioni, premesse sempre le debite emmiffioni di sangue, nell' empiema, nelle vomiche, nella cachessia, e forse ancora nell' Idrotorace, e Idrocardia. Che codesta radice dia robustezza ai solidi, io lo ripeto dal pronto ristabilimento delle forze in ogni caso

(7) L'aria di Mantova essendo di sua natura umida, dispone lentamente a quelle malattie, che nascono da vizio, e da lentore di linfa, e ciò principalmente per diminuzione d'irritabilità e di elaterio de'solidi. Le parti pri. me a soffrine $i$ tristi effetti sono $i$ visceri del basso ventre. Ier conseguenza un chilo mal preparato, vischioso, e crudo. si porta ai vasi del polmone, quali non essendo abbastanza robusti per digerirlo, e convertirlo in buon sangue, ne seguono degli arresti, o almeno rimane impedita la secrezione delle glandolette bronchiali, quindi le tossi, i catarri, i tubercoli, gl' infarcimenti, ed altre analoghe malattic di petto. 
da me osservato, ed a questa facoltà probabilmente si deve altresi la prontezza, con cui si sedarono il vomito, la colica, il singhiozzo (osserv. 4.). Se ciò è vero, quante non sarebbero le malattie, e le circostanze, nelle quali riescirebbe di pronto soccorso un rimedio semplice per se, uè punto ributtante, ma eguaimente efficace, e sollecito ne' suoi efferti? Rifletto inoltre, che il medesimo a prefirenza di altri moli, da quali abuonda anche di trop. po la Farmacia, si può praticare tanto ne' casi, ove sembra convenire un regime refrigerante, come nelle inframmazioni, nell' emofrici ec. ...., quanto in contrario nelle flussioni catarrali, nè ristagni di línfa, ne'mali finalmente sieno essi di acuta, o di cronica natura. Devo però avvertire, che volendosi praticare la Calaguala ne' mali acuti, pa$r e$ indispensabile una rigorosissima dieta: anzi quando il caso non apportasse una dilazione di più giorai, o l'ammalato per effetto d' 
estrema innarizione non esigesse qualcin sorta di nutrimato, sarei d'avviso, si dovesse istituire un' assoluta astinenza da cibi ne' cafi sing larmenre di esacerbata infiammazione, ò di orgasmo de' fluidi.

Se alle descricte particolarita della Calas guala aggiungasi, che la di lei azione si estende ad ogni età e temperamento, in ogni stagione, o clima, ed in qualunque possibile circostanza, la vedramo gareggiare negli effetti con la China-China, al pari di cui sopprimendo certe malattie senza crisi assai manifesta, opera in un mudo ann meno efficace che misterioso. Noi anderemo debitori all' America, oltre le molte altre produzioni, di un secondo tesoro, che potrebb' egualmente pretendere al tirolo di specifico altronde nella Terapeutica tanto desiderato.

Ma quali principj contiene l'esotica radice? Io non ho avuto il campo di analizzarla, nè so che altri l'abbia fatto. Se, co- 
me confido, ne potrò ottener quantità sufficiente, farò su di essa varie chimiche e fisiche esperienze. Intanto $\mathrm{mi}$ trovo in dovere di prevenire i Signori Speziali della Città e Stato, perche diansi ogni premura di arricchire le loro Farmacie di questa nuova Radice, e d' invitare i Clinici illustri a praticarla con quelle viste, che sono loro proprie. I risultati di molte, e reiterate osservazioni verranno a realizzare le facoltà del rimedio, perche, se vere, possa il Pubblico parteciparne, se false, venga disingannato.

\section{F I N E.}






$$
5858
$$


